\title{
TREATMENT ADHESION IN PATIENTS WITH SYSTEMIC SCLEROSIS AT AN OUTPATIENT RHEUMATOLOGY CLINIC IN A REFERRAL HOSPITAL
}

\begin{abstract}
Thiago Henrique da Silva Rached ${ }^{1}$, Rafael da Rocha Caminha ${ }^{1}$, Pedro Arturo Bismara Carneiro Santos ${ }^{1}$, Hugo Deleon de Lima ${ }^{1}$,

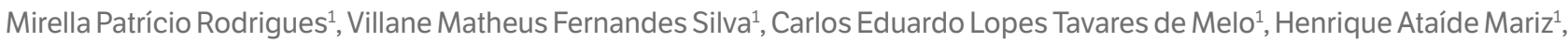
Laurindo Ferreira da Rocha Junior ${ }^{1, *}$
\end{abstract}

1.Instituto de Medicina Integral Professor Fernando Figueira, Recife (PE), Brazil.

*Corresponding author: laurindorochajr@hotmail.com

\section{BACKGROUND}

Adherence to treatment is a challenge in the management of chronic diseases. Few studies have evaluated drug adherence in systemic sclerosis (SSc). This study aimed to analyze medication compliance in patients with SSc at a tertiary hospital in the state of Pernambuco.

\section{METHODS}

A descriptive, cross-sectional study was performed in rheumatology outpatient clinic at Instituto de Medicina Integral Professor Fernando Figueira (IMIP). We included patients that fulfilled classification criteria for systemic sclerosis. Demographic and clinical characteristics were collected during visit consultations and by medical record reviews. Medication adherence was measured with the Morisky-Green-Levine scale and with the measurement of treatment adherence (MAT) questionnaire. GraphPad Prism V 6.01 was used to perform statistical analysis. A $p<0.05$ was considered statistically significant.

\section{RESULTS}

A total of 22 patients were included. One patient had not started the treatment prescribed and had to be excluded. Using the Morisky questionnaire, 6 (28.6\%) patients had low adherence, 10 (47.6\%) patients had medium adherence and 5 (23.8\%) patients had high adherence. Patients who forgot to supply their medications before finished had worse visual analogue scales (VAS) scores for Raynaud's phenomenon (34 IQR: 26-98) compared to those who did not forget (IQ: 0.0-53.5), $p=0.0252$. Morisky questionnaire showed negative correlation with VAS scores for digital ulcers $(r=-0.5026, p=0.0202)$. Using MAT questionnaire, $14(66.6 \%)$ patients were found to be adhering to their treatment. "Adherent" patients had significantly minor VAS scores for digital ulcers compared to "nonadherent patients", 1.5 (IQR: 0.0-40.5) vs. 49 (IQ: 4-95) respectively, $p<0.05$. Measurement of treatment adherence correlated negatively with VAS score for digital ulcers $(r=-0.6342, p=0.0020)$. "Adherent" patients had negative correlation of MAT with disease duration $(r=-0.6658, p=0.0131)$.

\section{CONCLUSION}

Adherence is a multifactorial phenomenon in patients with systemic sclerosis. A minority of the patients analyzed had high adherence to treatment using the Morisky questionnaire. Medication compliance is a very important factor that might increase therapeutic outcome in patients with systemic sclerosis and must be part of the approach of physicians who manage this condition.

\section{KEYWORDS}

Systemic sclerosis, Adherence, Treatment. 